\title{
Single or Double Bilateral Greater Occipital Nerve Blocks to manage Headache after Spinal Obstetric Anesthesia
}

\author{
Ahmed M Salem ${ }^{1 *}$, Ahmed E Salem ${ }^{2}$, Amr Sharaf Eldin ${ }^{3}$ and Ahmed M Hagras ${ }^{4}$ \\ ${ }^{1}$ Department of Anesthesiology \& ICU, Benha University, Egypt \\ ${ }^{2}$ Department of Anesthesiology \& ICU, Tanta University, Egypt \\ 3Department of Obstetrics \& Gynecology, Benha University, Egypt \\ 4Department of Obstetrics \& Gynecology, Tanta University, Egypt \\ Submission: March 25, 2019; Published: April 10, 2019 \\ *Corresponding author: Ahmed M Salem, Department of Anesthesiology \& ICU, Faculty of Medicine, Benha University, Egypt
}

\begin{abstract}
Objectives: To evaluate outcome of bilateral Greater Occipital Nerve Block (GONB) for parturient had Cesarean Section (CS) under Spinal Anesthesia (SA) for management of post-Dural puncture headache (PDPH).

Patients \& Methods: PDPH severity was graded using modified Lybecker classification as mild, moderate or severe. Mild PDPH was managed conservatively at home. Patients had moderate, severe headache or persistent mild PDPH within 3-day undertook bilateral distal GONB (2\% lidocaine and $0.5 \%$ bupivacaine; $1: 1$ mixture) at junction of medial third and lateral two-thirds of line joining External Occipital Protuberance (EOP) to mastoid process. Patients who had minimal or still had moderate improvement on distal block, received proximal GONB at 3-cm below and 1.5- $\mathrm{cm}$ lateral to EOP. Pain was scored using the numerical pain scale pre- and 3-hr post procedure, 1 and 4 weeks thereafter. Patients had minimal improvement on proximal block or recurred pain severity had Epidural Blood Patch (EBP).
\end{abstract}

Results: 119 women (19.4\%) developed PDPH; 87 had mild PDPH and received conservative management that resulted in 4-wk success rate of 56.4\%. Patients with moderate and severe and mild PDPH that failed to respond to conservative management undertook distal GONB that showed primary, 1-wk and 4-wk success rates of $82.9 \%, 64.3 \%$ and $61.4 \%$, respectively. 25 patients undertook proximal block for success rate of $78 \%$.

Conclusion: Bilateral GONB is simple, minimally invasive, safe and efficient therapeutic modality for moderate, severe and mild headache resistant to conservative treatment. Double, distal and proximal, block reduced the need for EBP down to $11.4 \%$.

Keywords: Spinal anesthesia; Cesarean section; Post dural punctures headache; Greater occipital nerve block; Single distal block; Double distal and proximal block

Abbreviations: GONB: Greater Occipital Nerve Block; CS: Cesarean Section; SA: Spinal Anesthesia; EOP: External Occipital Protuberance; NRS: Numeric Rating Scale; PDPH: Post-Dural Puncture Headache

\section{Introduction}

Guidelines for obstetric anesthesia recommend neuraxial anesthesia for Cesarean Section (CS) in most patients for being simple to perform, economical and produces rapid onset of anesthesia and complete muscle relaxation [1]. However, Spinal Anesthesia (SA) carries many hazards, where the incidence of maternal hypotension, without any prophylactic management, is about $80 \%$ [2] and about $60 \%$ of pregnant women had CS under SA suffer from Post-Dural Puncture Headache (PDPH) [3].

PDPH is defined as any headache occurring in the fronto-occipital area after a lumbar puncture that worsens within 15 minutes of sitting or standing and is relieved within 15 minutes of lying down [4]. PDPH is caused by excessive leakage of cerebrospinal fluid through the Dural puncture [5] and is distressing condition that may be debilitating and chronic [3].

Time of onset of PDPH was variable; $90 \%$ of headaches occur within 3 days of procedure [6] and two thirds within the first 48 hours, but very rarely, it can occur immediately after or develop up to 14 days of the procedure [7]. Fortunately, most cases of PDPH can resolve spontaneously without treatment within 7 days [8].

Prolonged bed rest [9], hydration and caffeine intake [10] and analgesia are commonly used as prophylaxis and treatment for PDPH [11]; however, no substantial evidence supports routine 
bed rest and aggressive hydration [12]. For patients with unsuccessful conservative PDPH management, the Epidural Blood Patch (EBP) is the most effective treatment option [13].

Peripheral nerve blocks are well tolerated and effective as adjunctive therapy for many disabling headache disorders [14]. Several studies suggested efficacy of Greater Occipital Nerve Block (GONB) in the treatment of migraine [15], cluster headache [16] and chronic daily headache [17]. Greater Occipital Nerve (GON) arises from C2-3 segments, its most proximal part lies between obliquus capitis inferior and semispinalis, near the spinous process. Then, GON enters into semispinalis passing through it and after its exit; it enters into trapezius muscle [18]. In distal region of trapezius fascia, it is crossed by the occipital artery and finally the nerve exits the trapezius fascia insertion into the nuchal line about 5-cm lateral to midline [19]. Functionally, GON supplies major rectus capitis posterior muscle, and the skin, muscles, and vessels of the scalp, but is the main sensory supply of occipital region [20].

\section{Objectives}

The current study aimed to evaluate the outcome of bilateral GON block (GONB) as a therapeutic modality for PDPH and to evaluate the outcome of double-bilateral block in resistant cases.

\section{Setting}

University and multiple private Hospitals in Benha and Tanta, Egypt

\section{Design}

Prospective clinical trial

\section{Patients \& Methods}

The study protocol was approved by the Local Ethical Committees and parturient and her husband signed written fully informed consents according to the declaration of Helsinki. All pregnant women assigned for CS for any indication other than fetal distress were eligible for evaluation. Women with history of previous meningitis, encephalitis, migraine, tension headache, cluster headache, neuralgia, hypertension, cerebral vascular accident, postpartum cerebral angiopathy, intracranial pathologies inducing intracranial hypertension, dehydration and caffeine withdrawal were excluded from the study. Also, women who had pre-eclampsia, vertebral column anomalies or skin, renal, hepatic or cardiac diseases and blood diathesis were also excluded from the study.

All parturient were clinically examined for indication of CS and fetal condition, and then were clinically examined for ASA grade, presence of exclusion criteria and spine was also examined. Patients attended the theater fasting at least 6 hours and without administration of pre-medications or preoperative intravenous fluids. Spinal anesthesia was conducted using $10 \mathrm{mg}$ bupivacaine $0.5 \%$ injected through 25G Quincke needle with a medium bevel cutting inserted in the L3-4 or L 4-5 interspace. Spinal anesthesia was done in sitting position then the parturient was asked to lie down immediately after bupivacaine injection. Intravenous fluid was given in a dose of $15 \mathrm{ml} / \mathrm{kg}$ warm lactated ringer solution started as fast drip during and continued after spinal anesthesia. Systolic Blood Pressure (SBP) was non-invasively monitored for development of hypotension; defined as SBP $<100 \mathrm{mmHg}$ and was treated with ephedrine.

Patients were managed as day-case and allowed to return home after complete resolution of sensory and motor block, and stability of SBP at level of $>100 \mathrm{mmHg}$. Patients were asked to follow lines of conservative management; bed rest, not to get upright position suddenly, receive plenty of fluids and mild oral analgesia and to notify by phone on development of headache of any severity or other manifestations of PDPH; namely, nausea, vomiting, dizziness, tinnitus or sense of deafness. For comparative purposes, enrollment data of patients who did not develop manifestations of PDPH were collected as control group.

\section{PDPH assessment and grading}

Patients developed headache or other manifestations of PDPH were asked to attend the hospital for evaluation and management. Headache severity was evaluated using a modified Lybecker classification [21] into: Mild inducing slight restriction of daily activities, but patient was not bedridden and had no associated symptoms and headache responds well to oral analgesics. Moderate headache is that inducing significant restriction of daily activities and patient was bedridden most of the day, but it may or not be associated other symptoms and pain required injectable analgesia. Severe PDPH is that induces complete restriction of daily activities, patient is bedridden all day and associated symptoms are present. Patients with mild PDPH were returned home and asked to continue conservative management for three days aiming for spontaneous resolution otherwise to return to hospital. All patients with moderate and severe PDPH, mild PDPH that failed to resolve within 3-day conservative treatment underwent bilateral GONB

\section{GONB procedure}

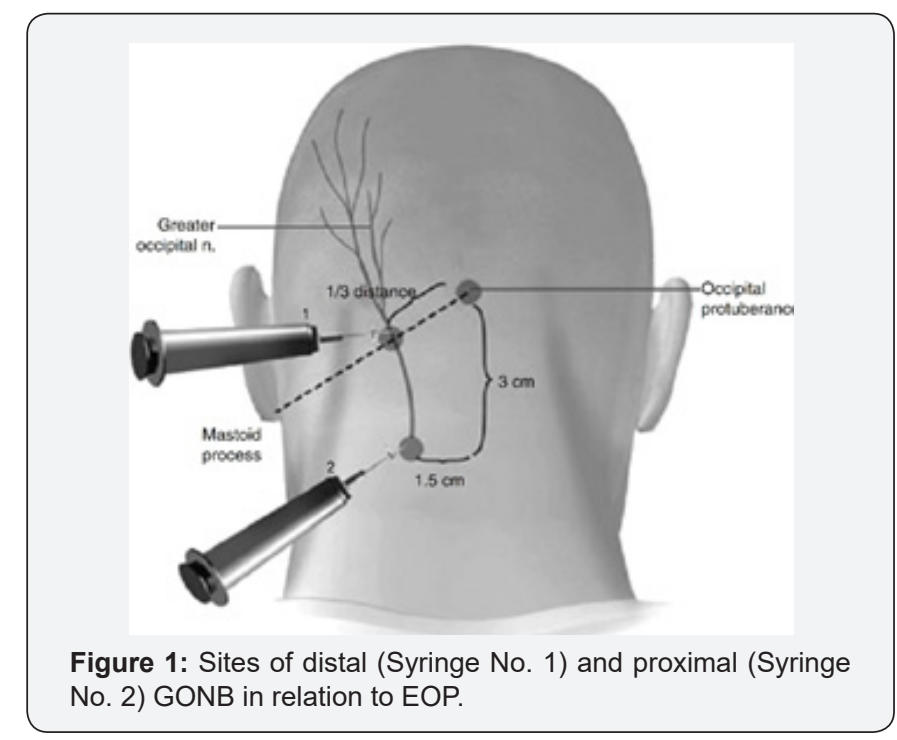


GONB was performed as described by Young et al. [22] using 3 -ml of a 1:1 mixture of lidocaine (2\%) and bupivacaine $(0.5 \%)$ injected by 3-ml syringe with a 25-30-gauge, 1-1.5 in. needle. Briefly, with the patient in setting position and neck was flexed, the occipital artery was localized at the junction of medial third and lateral two-thirds of a line draw between the External Occipital Protuberance (EOP) and the mastoid process (Figure 1), and the GON was localized often just medial to the artery in a region where no muscle is present $[22,23]$. Palpating this region often elicited tenderness, which confirms the correct location for the injection, and $1.5 \mathrm{ml}$ of anesthetic mixture was injected (Distal injection technique; Syringe no. 1 in Figure 1). Then, the procedure was repeated on the opposite site to achieve bilateral block.

Patients were assessed for pain using an 11-point Numeric Rating Scale (NRS) with numbers from 0 to 10 where 0 indicates no pain and 10 indicates worst pain imaginable [24,25]. Pain score was assessed pre-procedural and 1-hourly for three hours; improvement was quantified based on the percentage change from baseline NRS score as significant on $>50 \%$ NRS score reduction, moderate on NRS score reduction by $31-50 \%$, or minimal on $<30 \%$ NRS score reduction [26]. Patients documented significant and moderate improvement were allowed to return home, while patients had minimal improvement received proximal GONB, where the anesthetic fluid was injected at exit of GON from semispinalis muscle, $3-\mathrm{cm}$ below and $1.5-\mathrm{cm}$ lateral to the EOP (Proximal injection technique; Syringe no. 2 in Figure 1) and were allowed to return home. Pain scores were collected again at 1 and 4 weeks after the procedure and patients who had single block and still having moderate improvement undertook proximal block. Patients had minimal improvement on the proximal block

Table 1: Patients' enrolment data.

\begin{tabular}{|c|c|c|c|c|c|}
\hline \multicolumn{3}{|c|}{ Group Data } & Control (No PDPH Group; $n=493$ ) & Study (PDPH Group; n=119) & P value \\
\hline \multicolumn{3}{|c|}{ Age (years) } & $28.5 \pm 2.2$ & $27.5 \pm 2.3$ & 0.011 \\
\hline \multirow{3}{*}{ BMI data } & \multicolumn{2}{|c|}{ Body weight $(\mathrm{kg})$} & $87.9 \pm 7.6$ & $90.7 \pm 5.7$ & 0.007 \\
\hline & \multicolumn{2}{|c|}{ Body height $(\mathrm{cm})$} & $164.1 \pm 3.2$ & $164.6 \pm 3.1$ & 0.269 \\
\hline & \multicolumn{2}{|c|}{ BMI $\left(\mathrm{kg} / \mathrm{m}^{2}\right)$} & $32.7 \pm 2.9$ & $33.5 \pm 2.5$ & 0.047 \\
\hline \multirow{4}{*}{ Parity } & \multicolumn{2}{|c|}{ Primi } & $302(61.2 \%)$ & $82(68.9 \%)$ & \multirow{4}{*}{0.639} \\
\hline & \multirow{3}{*}{ Multi } & 1 offspring & $96(19.5 \%)$ & $21(17.6 \%)$ & \\
\hline & & 2 offspring & $61(12.4 \%)$ & $11(9.2 \%)$ & \\
\hline & & 3 offspring & $34(6.9 \%)$ & $5(4.3 \%)$ & \\
\hline \multicolumn{3}{|c|}{ Gestational age (wk) } & $38 \pm 0.7$ & $38.2 \pm 0.9$ & 0.188 \\
\hline \multirow{2}{*}{ ASA grade } & & I & $288(86 \%)$ & $73(89 \%)$ & \multirow{2}{*}{0.298} \\
\hline & & II & $47(14 \%)$ & $9(11 \%)$ & \\
\hline \multirow{5}{*}{ Indication for elective CS } & Abnor & placental site & $162(32.8 \%)$ & $41(34.5 \%)$ & \multirow{5}{*}{0.783} \\
\hline & & ormal lie & $123(24.9 \%)$ & $32(26.9 \%)$ & \\
\hline & & icted pelvis & $38(7.7 \%)$ & $16(13.4 \%)$ & \\
\hline & & vious CS & $116(23.5 \%)$ & 23 (19.3\%) & \\
\hline & & thers & $54(11 \%)$ & 7 (5.9\%) & \\
\hline
\end{tabular}

Data are presented as mean \pm SD, numbers \& percentages; PDPH: Post-dural puncture headache; BMI: Body mass index; CS: Cesarean section; $p$ value $>0.05$ indicates non-significant difference, $p$ value $<0.05$ indicates significant difference between both groups.

or recurred pain severity during follow-up were shifted to have epidural blood patch

\section{Study outcomes}

a) Frequency of PDPH after spinal anesthesia for CS using 25G Quincke needle with a medium bevel cutting

b) The analgesic outcome of GONB at end of 4-wk follow-up

c) The frequency of proximal block and EBP to manage residual or persistent PDPH.

\section{Sample size calculation}

Review of literature showed that frequency of PDPH on using 25G Quincke needle with a medium bevel cutting is 36\% [27] but its incidence obtained by Jabbari et al. [28] was $17.3 \%$, to get study power of $80 \%$ with $\alpha$ value of 0.05 and $\beta$ value of 0.2 , sample size per group was calculated to be $>50$ patients per group.

\section{Statistical analysis}

Obtained data were presented as mean $\pm \mathrm{SD}$, numbers and percentages. Results were analyzed using paired t-test, One-way ANOVA Test and Chi square test. Statistical analysis was conducted using the IBM SPSS (Version 23, 2015) for Windows statistical package. $P$ value $<0.05$ was considered statistically significant.

Results

During 4-year duration, 673 parturient assigned to CS were eligible for evaluation; 45 women were excluded for not fulfilling the inclusion criteria and 628 women were included in the study. Sixteen women had failed SA and were excluded from the study and 612 women had CS under SA without the need to shift to general anesthesia. 
One hundred and nineteen women developed PDPH for a frequency of $19.4 \%$ and were included as Study group, while the remaining 493 women were considered as control group. Parturient of study group were significantly younger and had significant- ly lower body weight and body mass index. However, the other enrolment data showed non-significant differences between patients of both groups (Table 1).

Table 2: Hypotension data.

\begin{tabular}{|c|c|c|c|c|}
\hline & & $\begin{array}{c}\text { Control Group } \\
\text { (No PDPH; n=493) }\end{array}$ & $\begin{array}{c}\text { Study Group } \\
\text { (PDPH; n=119) }\end{array}$ & P Value \\
\hline \multirow{2}{*}{ SBP (mmHg) } & Baseline & $119.1 \pm 4.5$ & $119.7 \pm 4.7$ & 0.413 \\
\hline & Lowest level & $86.6 \pm 3.8$ & $85.8 \pm 8.7$ & 0.520 \\
\hline \multirow{4}{*}{ Hypotension data } & Frequency & $140(28.4 \%)$ & $41(34.5 \%)$ & 0.387 \\
\hline & Time since SA till SBP is $<100 \mathrm{mmHg}(\mathrm{min})$ & $8.4 \pm 2.2$ & $7.9 \pm 1.6$ & 0.293 \\
\hline & Duration of hypotension (min) & $7.7 \pm 3.9$ & $8.3 \pm 2.7$ & 0.169 \\
\hline & Dose of ephedrine used (mg) & $12.3 \pm 3.8$ & $13.7 \pm 3.7$ & 0.084 \\
\hline
\end{tabular}

Data are presented as mean \pm SD, numbers \& percentages; PDPH: Post-dural puncture headache; SBP: Systolic blood pressure; SA: Spinal anesthesia; $p$ value $>0.05$ indicates non-significant difference between both groups; $p$ value $<0.05$ indicates significant difference between both groups.

Hypotension with SBP $<100 \mathrm{mmHg}$ was recorded in 225 patients $(36.8 \%)$ with non-significant $(\mathrm{p}>0.05)$ difference between both groups regarding time since SA till development of hypotension, the lowest level of SBP, duration of hypotension, and dose of ephedrine required to combat hypotension (Table 2).

According to modified Lybecker classification; 28 women (23.5\%) had moderate and 4 women (3.4\%) had severe PDPH, these 32 patients undertook distal GONB. The remaining 87 women $(73.1 \%)$ had mild PDPH and were allowed to return home, were maintained on conservative management and give daily comment by phone. At 1-wk follow-up, 23 patients were still complaining of headache that limited their daily activities and undertook distal block, while the remaining 64 patients were good, and their headache had resolved. Unfortunately, 15 of these 64 patients developed recurrent headache after resolution and received distal block. Collectively, 49 patients had 4-wk complete headache resolution on conservative treatment for a success rate of $56.3 \%$.

Seventy women undertook distal GONB and at end of 3-hr follow-up, 41 women had significant improvement, 17 had moderate and 12 had minimal improvement. These 12 patients undertook proximal block, while the remaining 58 patients were allowed to return home and re-attend the hospital at one and four weeks for follow-up. At 1-wk visit, 45 patients had significant improvement, while 13 were still having moderate improvement and undertook proximal block. At 4-wk visit, 43 patients had complete resolution for a success rate of distal block of $61.4 \%$; two patients developed deterioration of their improvement and undertook EBP.

Twenty-five patients undertook proximal block that resulted in significant and moderate improvement in 18 and 4 patients, respectively and were allowed to return home and re-attend for 1-wk and 4-wk follow-up; while the remaining 3 patients had mild improvement and undertook EBP. At 1-wk, all of the 22 patients had progressive improvement, but at 4 -wk visit 19 patients had complete resolution of headache for success rate of $78 \%$ for double-block procedure, while three patients were still complaining and were shifted to EBP. Thus, 8 patients required EBP for management of their headache and were considered as failure of GONB for a rate of $11.4 \%$.

\section{Discussion}

Acute headache after CS under SA is still up till now a problem, and hormonal, vascular, and procedural factors elevate its risk and absence of headache history and clear PDPH description should prompt strong consideration for evaluation and management [29], so this study tried to evaluate outcome of GONB as a minimally invasive therapeutic modality for parturient had CS under SA and developed PDPH.

Women developed PDPH were significantly younger and had significantly lower BMI than those passed their parturition free of PDPH. These findings spot light on a possible role for patients' age, BMI and vasomotor tone insatiability and development of PDPH and go in hand with Peralta et al. [30] \& Franz et al. [31] who detected decreased PDPH incidence after Dural puncture in parturient with an increased BMI. Also, Bakshi \& Gehdoo [32] and Özcan et al. [33] detected a relation between incidence of PDPH and parturient age and found incidence was higher in age group of 20-40 years compared to older patients.

Patients had mild PDPH ( $\mathrm{n}=87)$ received conservative management and follow-up, aiming for headache resolution, depending on the previous knowledge that PDPH can be self-limiting condition within 7-days [8]. Unfortunately, at 1-wk 23 patients had persistent headache and at 4-wk, 15 patients had recurrent headache and only 49 patients reported 4-wk complete resolution of PDPH for a success rate of $56.4 \%$. These findings point to a fact that PDPH is not solely self-limiting condition and necessitate follow-up even for cases with mild manifestations. In line with this assumption, Gauthama et al. [34] reported that at 18 months after the procedure, PDPH persisted in 30\%, worsened in $15 \%$ of patients and another $15 \%$ of patients developed new onset headache, so concluded that PDPH can no longer be considered a self-limiting condition.

Patients with moderate $(n=28)$ and severe $(n=4)$ and those had mild PDPH but failed to respond to conservative management 
$(n=38)$ undertook distal GONB and followed for one and 4 weeks after procedure, and those failed to respond had proximal GOBN and followed similarly. The rational for follow-up for this duration aiming for resolution of headache go in hand with Lambru et al. [17] who considered responders to GONB as patients with a complete or partial response lasting at least 7 days and reported duration of a positive response ranged between 7-504 days. Also, Zipfel et al. [35] used GONB for management of varied types of headache and reported clinical efficacy, defined as decrease of at least $50 \%$ of visual analog scores, of $80 \%, 66.7 \%$ and $60 \%$ of cases at 7 days, one month and 3 months, respectively.

At 3-hr after distal GONB, 58 patients reported moderate-to-significant improvement, for primary success rate of $82.9 \%$, and at 1 -wk and 4-wk follow-up, the success rates were $64.3 \%$ and $61.4 \%$, respectively. The reported primary success rate was superior to that reported by Niraj et al. [36] who found headache resolved in $66 \%$ of patients received GONB. Twenty-five patients undertook proximal block for minimal improvement on distal block ( $\mathrm{n}=12)$ and persistence of moderate improvement after distal block for 1 -wk, and at 4-wk visit 19 patients had complete resolution of headache for success rate of $78 \%$.

In line with double-block for GON for PDPH management, Uyar Türkyilmaz et al. [37] used levobupivacaine and dexamethasone mixture for GONB in 18 women had post-CS PDPH and reported significantly decreasing VAS pain scores gradually and VAS score was $<3$ at the end of 24 -hr after injection in all patients, but on 2 nd day, 6 patients had increased pain intensity and in two patients VAS score was $>3$ and received another block, but one failed to respond and had EBP. However, these results can be critiqued for only immediate evaluation, the rapid decision for second block-setting and the repeated block at the same site.

The beneficial effect of the second-block can be attributed to tender or triggering point block because proximal GONB entails nerve block while it traverses the semispinalis muscle to the trapizus muscle, so proximal nerve injection induces nerve block and anesthetizes the muscles which could be the headache trigger point. In support of this assumption, Nystrom \& Freeman [38] reported that peripheral pain thresholds can be modulated by manipulations at myofascial tender points especially in patients with central sensitization expressed as lower pain thresholds and suggested surgical ablation of these points as treatment modality for central sensitization.

PDPH is characterized by fronto-occipital headache [4], so it is predicted for blocking of GON, which is the main sensory supply of occipital region [20], to allow relieve of occipital pain, while its relieving effect of frontal headache could be explained by its communications with the trigeminal nerve nucleus and 3rd occipital nerve. In support of this assumption, Baek et al. [39], out of their cadaver study, reported that the clinical efficacy of GONB seems unlikely to arise from the blockade of GON alone, but it likely arises from blockade of most nerves originating from the dorsal ramus of the upper cervical spinal nerve at the suboccipital area; namely the suboccipital, third occipital and greater occipital nerves. Also, García-Magro et al. [40] reported convergence of cervical and trigeminal afferents on the same territories of the upper cervical and lower medullary dorsal horn (The trigeminocervical complex) and in animal model of migraine found GON block or stimulation alter nociceptive processing in trigeminocervical complex.

Only 8 patients required EBP for management of their headache and were considered as failure of GONB for a rate of $11.4 \%$. The EBP-sparing effect of double-GONB was superior to that reported by Niraj et al. [36] who documented the need for EBP in $34 \%$ of their PDPH patients after failure of single-GONB. Multiple previous studies $[3,41,42]$ assured the efficacy of EBP as the gold standard in treating PDPH; however, it is invasive procedure with questionable cost-effectiveness and documented risk of dural puncture, neurologic complications, bleeding, and infection [43]. On the other side, the applied GONB procedure reduced the need for EBP down to 11.4\% among patients had PDPH, and being minimally invasive, can be performed on outpatient basis and its minimal cost could be considered as additional favor for such patients. In support of this assumption, Nair et al. [20] documented that bilateral GONB is minimally invasive and simple procedure for management of chronic headache in patients with PDPH and allows avoidance of EBP.

\section{Conclusion}

Bilateral GONB is simple, minimally invasive, safe and efficient therapeutic modality for patients developed PDPH after SA for CS. GONB can manage moderate, severe and mild headache resistant to conservative treatment. Double, distal and proximal, block reduced the need for EBP down to 11.4\%, irrespective of headache severity.

\section{References}

1. Cobb BT, Lane Fall MB, Month RC, Onuoha OC, Srinivas SK, et al. (2018) Anesthesiologist Specialization and Use of General Anesthesia for Cesarean Delivery. Anesthesiology 130(2): 237-246.

2. Ryu C, Choi GJ, Park YH, Kang H (2019) Vasopressors for the management of maternal hypotension during cesarean section under spinal anesthesia: A Systematic review and network meta-analysis protocol. Medicine (Baltimore) 98(1): e13947.

3. Ioscovich A, Giladi Y, Fuica RL, Weiniger CF, Orbach Zinger S, et al. (2018) Anesthetic approach to postdural puncture headache in the peripartum period: An Israeli national survey. Acta Anaesthesiol Scand 62(10): 1460-1465.

4. Ravn A, Lyckhage LF, Jensen R (2018) Post-dural puncture headache. Ugeskr Laeger 180(20).

5. Arevalo Rodriguez I, Muñoz L, Godoy Casasbuenas N, Ciapponi A, Arevalo JJ, et al. (1993) Needle gauge and tip designs for preventing post-dural puncture headache (PDPH). Cochrane Database Syst Rev 4: CD010807.

6. Reynolds F (1993) Dural puncture and headache. Br Med J 306: 874876.

7. Leibold RA, Yealy DM, Coppola M, Cantees KK (1993) Post-dural puncture headache: Characteristics, management, and prevention. Ann Emerg Med 22(12): 1863-1870. 
8. Costigan SN, Sprigge JS (1996) Dural puncture: the patient's perspective. A patient survey of cases at a DGH maternity unit 1983-1993. Acta Anaesth Scand 40(6): 710-714.

9. Arevalo Rodriguez I, Ciapponi A, Roqué Figuls M, Muñoz L, Bonfill Cosp $X$ (2013) Posture and fluids for preventing post-dural puncture headache. Cochrane Database Syst Rev 12(7): CD009199.

10. D'souza G, Seidel FG, Krane EJ (2017) Management of a Ventral Cerebrospinal Fluid Leak with a Lumbar Transforaminal Epidural Blood Patch in a Child with Persistent Postdural Puncture Headache: A Case Report. Reg Anesth Pain Med 42(2): 263-266.

11. Tien JC, Lim MJ, Leong WL, Lew E (2016) Nine-year audit of post-dural puncture headache in a tertiary obstetric hospital in Singapore. Int J Obstet Anesth 28: 34-38.

12. Kwak KH (2017) Postdural puncture headache. Korean J Anesthesiol 70(2): 136-143.

13. Xu H, Liu Y, Song W, Kan S, Liu F, et al. (2017) Comparison of cutting and pencil-point spinal needle in spinal anesthesia regarding postdural puncture headache: A meta-analysis. Medicine (Baltimore) 96(14): e6527.

14. Lambrinakos Raymond K, Dubrovsky AS (2018) Nerve blocks in paediatric and adolescent headache disorders. Curr Opin Pediatr 30(6): 780-785.

15. Özer D, Bölük C, Türk Börü Ü, Altun D, Tașdemir M, et al. (2018) Greater occipital and supraorbital nerve blockade for the preventive treatment of migraine: a single-blind, randomized, placebo-controlled study. Curr Med Res Opin 35(5): 909-915.

16. Wei J, Robbins MS (2018) Greater Occipital Nerve Injection versus Oral Steroids for Short Term Prophylaxis of Cluster Headache: A Retrospective Comparative Study. Headache 58(6): 852-858.

17. Lambru G, Abu Bakar N, Stahlhut L, McCulloch S, Miller S, et al. (2014) Greater occipital nerve blocks in chronic cluster headache: a prospective open-label study. Eur J Neurol 21(2): 338-343.

18. Janis JE, Hatef DA, Ducic I, Reece EM, Hamawy AH, et al. (2010) The anatomy of the greater occipital nerve: Part II. Compression point topography. Plast Reconstr Surg 126(5): 1563-1572.

19. Güvençer M, Akyer P, Sayhan S, Tetik S (2011) The importance of the greater occipital nerve in the occipital and the suboccipital region for nerve blockade and surgical approaches--an anatomic study on cadavers. Clin Neurol Neurosurg 113(4): 289-294.

20. Nair AS (2018) Bilateral greater orbital nerve block: Efficacy in postdural puncture headache. Saudi J Anaesth 12(1): 159-160.

21. Lybecker H, Djernes M, Schmidt JF (1995) Postdural puncture headache (PDPH): onset, duration, severity, and associated symptoms. An analysis of 75 consecutive patients with PDPH. Acta Anaesthesiol Scand 39(5): 605-612.

22. Young WB, Marmura M, Ashkenazi A, Evans RW (2008) Expert opinion: greater occipital nerve and other anesthetic injections for primaryheadache disorders. Headache 48(7): 1122-1125.

23. Mosser SW, Guyuron B, Janis JE, Rohrich RJ (2004) The anatomy of the greater occipital nerve: implications for the etiology of migraine headaches. Plast Reconstr Surg 113(2): 693-700.

24. Williamson A, Hoggart B (2005) Pain: a review of three commonly used pain rating scales. J Clin Nurs 14(7): 798-804.

25. Fairbank JC, Couper J, Davies JB, O Brien JP (1980) The oswestry low back pain disability questionnaire. Physiotherapy 66(8): 271-273.

26. Childs JD, Fritz JM, Flynn TW (2005) Treatments for back pain. Ann Intern Med 142(10): 874.

27. Kuntz KM, Kokmen E, Stevens JC, Offord KP, Ho MM (1992) Post lumbar puncture headache: experience in 501 consectuve procedure. Neurology $42(10): 1884-1887$

28. Jabbari A, Alijanpour E, Mir M, Bani Hashem N, Rabiea SM, et al. (2013) Post spinal puncture headache, an old problem and new concepts: review of articles about predisposing factors. Caspian J Intern Med 4(1): 595-602.

29. Vgontzas A, Robbins MS (2018) A Hospital Based Retrospective Study of Acute Postpartum Headache. Headache 58(6): 845-851.

30. Peralta F, Higgins N, Lange E, Wong CA, McCarthy RJ (2015) The Relationship of Body Mass Index with the Incidence of Postdural Puncture Headache in Parturients. Anesth Analg 121(2): 451-456.

31. Franz AM, Jia SY, Bahnson HT, Goel A, Habib AS (2017) The effect of second-stage pushing and body mass index on postdural puncture headache. J Clin Anesth 37: 77-81.

32. Bakshi SG, Gehdoo RSP (2018) Incidence and management of post-dural puncture headache following spinal anaesthesia and accidental dural puncture from a non-obstetric hospital: A retrospective analysis. Indian J Anaesth 62(11): 881-886.

33. Özcan Hİ, Tümtürk P, Pirbudak L (2019) Postdural puncture headache: Incidence and predisposing factors in a university hospital. Agri 31(1): 1-8.

34. Gauthama P, Kelkar A, Basar SMA, Niraj G (2019) Incidence of Persistent Headache at 18 Months Following Accidental Dural Puncture in the Obstetric Population: A Prospective Service Evaluation in $45 \mathrm{~Pa}-$ tients. Headache 59(1): 97-103.

35.Zipfel J, Kastler A, Tatu L, Behr J, Kechidi R, et al. (2016) Ultrasound-Guided Intermediate Site Greater Occipital Nerve Infiltration: A Technical Feasibility Study. Pain Physician 19(7): E1027-E1034.

36. Niraj G, Kelkar A, Girotra V (2014) Greater occipital nerve block for postdural puncture headache (PDPH): a prospective audit of a modified guideline for the management of PDPH and review of the literature. J Clin Anesth 26(7): 539-544.

37. Uyar Türkyilmaz E, Camgöz Eryilmaz N, Aydin Güzey N, Moraloğlu Ö (2016) Bilateral greater occipital nerve block for treatment of post-dural puncture headache after caesarean operations. Braz J Anesthesiol 66(5): 445-450.

38. Nystrom NA, Freeman MD (2018) Central sensitization is modulated following trigger point anesthetization in patients with chronic pain from whiplash trauma. A Double-Blind, Placebo-Controlled, Crossover Study. Pain Med 19(1): 124-129.

39. Baek IC, Park K, Kim TL, O J, Yang HM, et al. (2018) Comparing the injectate spread and nerve involvement between different injectate volumes for ultrasound-guided greater occipital nerve block at the $\mathrm{C} 2$ level: a cadaveric evaluation. J Pain Res 11: 2033-2038.

40. García Magro N, Martin YB, Negredo P, Avendaño C (2018) The greater occipital nerve and its spinal and brainstem afferent projections: A stereological and tract-tracing study in the rat. J Comp Neurol 526(18): 3000-3019.

41. Simopoulos TT, Sharma S, Aner M, Gill JS (2016) The Incidence and Management of Postdural Puncture Headache in Patients Undergoing Percutaneous Lead Placement for Spinal Cord Stimulation. Neuromodulation 19(7): 738-743.

42. Martínez B, Canser E, Alonso A, Alonso E, Gredilla E, et al. (2018) Postdural Puncture Headache and Epidural Blood Patch in a Large Obstetric Anaesthesia Population. Asian J Anesthesiol 56(1): 23-32.

43. Kent S, Mehaffey G (2016) Transnasal sphenopalatine ganglion block for the treatment of postdural puncture headache in obstetric patients. J Clin Anesth 34: 194-196. 
Your next submission with Juniper Publishers will reach you the below assets

- Quality Editorial service

- Swift Peer Review

- Reprints availability

- E-prints Service

- Manuscript Podcast for convenient understanding

- Global attainment for your research

- Manuscript accessibility in different formats ( Pdf, E-pub, Full Text, Audio)

- Unceasing customer service

Track the below URL for one-step submission https://juniperpublishers.com/online-submission.php 